\title{
Spectral-Element Moment Tensor Inversions for Earthquakes in Southern California
}

\author{
by Qinya Liu, Jascha Polet, Dimitri Komatitsch,* and Jeroen Tromp
}

\begin{abstract}
We have developed and implemented an automated moment tensor inversion procedure to determine source parameters for southern California earthquakes. The method is based upon spectral-element simulations of regional seismic wave propagation in an integrated 3D southern California velocity model. Sensitivity to source parameters is determined by numerically calculating the Fréchet derivatives required for the moment tensor inversion. We minimize a waveform misfit function, and allow limited time shifts between data and corresponding synthetics to accommodate additional 3D heterogeneity not included in our model. The technique is applied to three recent southern California earthquakes: the 9 September 2001, $M_{\mathrm{L}}$ 4.2 Hollywood event, the 22 February 2003, $M_{\mathrm{L}} 5.4$ Big Bear event, and the 14 December 2001, $M_{\mathrm{L}} 4.0$ Diamond Bar event. Using about half of the available threecomponent data at periods of $6 \mathrm{sec}$ and longer, we obtain focal mechanisms, depths, and moment magnitudes that are generally in good agreement with estimates based upon traditional body-wave and surface-wave inversions.
\end{abstract}

\section{Introduction}

Southern California is characterized by geologic and tectonic complexity. Every year about 40 earthquakes with local magnitude greater than 3.5 occur on the fault systems in the region, and the determination of the source parameters of these earthquakes is important for understanding the associated tectonic processes. Southern California Seismic Network (SCSN) first-motion data can be used to determine focal mechanisms of earthquakes (Hauksson et al., 2002). Regional long-period (10-50 sec) surface waves have been used to invert for source parameters by correcting for the effects of 3D heterogeneity with a regional phase-velocity map (Thio and Kanamori, 1995). However, in general this method is only suitable for earthquakes with $M_{\mathrm{W}} \geq 3.7$, because the signal-to-noise ratio becomes too low for smaller earthquakes at such long periods. Regional broadband waveform data have also been used to determine source parameters. The early part of the $P$ wave-that is, $P_{\mathrm{nl}}$, the combination of the $P_{\mathrm{n}}$ and $P L$ phases (Helmberger and Engen, 1980) — on the vertical and radial components of the seismograms is relatively insensitive to crustal heterogeneity. For this reason, this phase has been used to determine the strike, dip, and rake of moderate-size earthquakes (Wallace and Helmberger, 1981). Grid searching over strike, dip, rake, and depth was introduced to deal with the nonlinearity as-

\footnotetext{
*Present address: Geophysical Imaging Laboratory, CNRS FRE 2639, Université de Pau et des Pays de l'Adour, Avenue de l'Université, 64013 Pau Cedex, France.
}

sociated with these source parameters for southern California earthquakes (Dreger and Helmberger, 1991) based upon the 1D standard southern California velocity model (Hadley and Kanamori, 1977; Dreger and Helmberger, 1990). In order to utilize the whole waveform and accommodate the imperfect 1D Green's function, the vertical and radial components of the seismograms at local and regional distances are cut into $P_{\mathrm{nl}}$ and surface-wave segments. By allowing for time shifts between these two segments, source mechanisms can be resolved by fitting them simultaneously (Zhao and Helmberger 1994; Zhu and Helmberger, 1996).

Many more high-quality stations have been deployed by the SCSN since the early 1990s. A large portion of these are located in or near the Los Angeles basin, where complicated 3D velocity structure produces complicated waveforms. Simple time shifts do not adequately accommodate the imperfect 1D Green's function in the source inversions, and 3D Green's functions are required for a significant portion of the SCSN stations. For this purpose, this article introduces an adaptation of the centroid-moment tensor formalism (Gilbert and Dziewonski, 1975; Dziewonski et al., 1981; Ritsema and Lay, 1993) to determine the source mechanisms of small- to moderate-size earthquakes in southern California. The calculation of synthetic seismograms and Fréchet derivatives (derivatives of the synthetics with respect to source parameters) is accomplished based upon the spectralelement method (e.g., Komatitsch and Vilotte, 1998; Komatitsch and Tromp, 1999) and recently developed 3D 
southern California crustal velocity models (Hauksson, 2000; Süss and Shaw, 2003).

\section{Model Description}

We use an integrated crustal velocity model of southern California. We adopt the Hauksson (2000) tomographic model as the regional background model. Within this model we embed a recent Los Angeles basin $P$-velocity model developed by Süss and Shaw (2003), which extends from $119.3^{\circ} \mathrm{W}$ to $117.3^{\circ} \mathrm{W}$ and from $33.0^{\circ} \mathrm{N}$ to $34.8^{\circ} \mathrm{N}$. This model further contains a higher-resolution block within the Los Angeles basin, extending from $118.4^{\circ} \mathrm{W}$ to $117.9^{\circ} \mathrm{W}$ and from $33.7^{\circ} \mathrm{N}$ to $34.1^{\circ} \mathrm{N}$. This model was constructed in GOCAD, a 3D structural modeling tool (Mallet, 1992), and contains a detailed description of the sedimentary basement shape determined from hundreds of petroleum industry well logs and more than $20,000 \mathrm{~km}$ of seismic reflection profiles. We scale the $P$ velocity of the model through an empirical relation (Komatitsch et al., 2004) to obtain the shear velocity. The mesh for this integrated model follows the shape of the Moho determined by Zhu and Kanamori (2000) and topography and bathymetry obtained from the U.S. Geological Survey (Komatitsch et al., 2004). Figure 1a shows the integrated model in map view, and Figure $1 \mathrm{~b}$ shows $P$-velocity variations in two cross sections, $\mathrm{AA}^{\prime}$ and $\mathrm{BB}^{\prime}$, through the model. Cross section $\mathrm{AA}^{\prime}$ starts from the Coast Ranges, goes through the Ventura and Los Angeles basins, and ends in the Peninsular Ranges. As seen clearly in Figure 1b, both basins have very slow $P$ velocities (warm colors) due to the thick sediments. Cross section $\mathrm{BB}^{\prime}$ starts north of the east California shear zone, goes through the Mojave Desert, the San Gabriel Mountains, and the Los Angeles basin, and ends at the continental borderland. In the $P$-velocity cross section, the Los Angeles basin again appears with distinctly slow $P$ velocities at the surface. Notice the significant variations in surface topography, the difference in elevation between the San Gabriel Mountains and the continental borderland being about $3 \mathrm{~km}$. The Moho depth in southern California also varies significantly, from about $20 \mathrm{~km}$ underneath the continental borderland to roughly $40 \mathrm{~km}$ underneath the San Bernardino Mountains (Zhu and Kanamori, 2000).

This integrated model involves substantial 3D variations, precluding the use of simple quasi-analytic solutions or perturbation theory. The accurate simulation of seismic wave propagation in this complicated 3D model is crucial to minimize the effects of numerical imperfections on the inversion for source parameters. Therefore, we use the recently developed spectral-element method to simulate wave propagation in this integrated 3D velocity model.

\section{Spectral-Element Method}

The spectral-element method has been used extensively to simulate seismic wave propagation on both global and regional scales (e.g., Komatitsch and Tromp, 2002a,b; Chal-

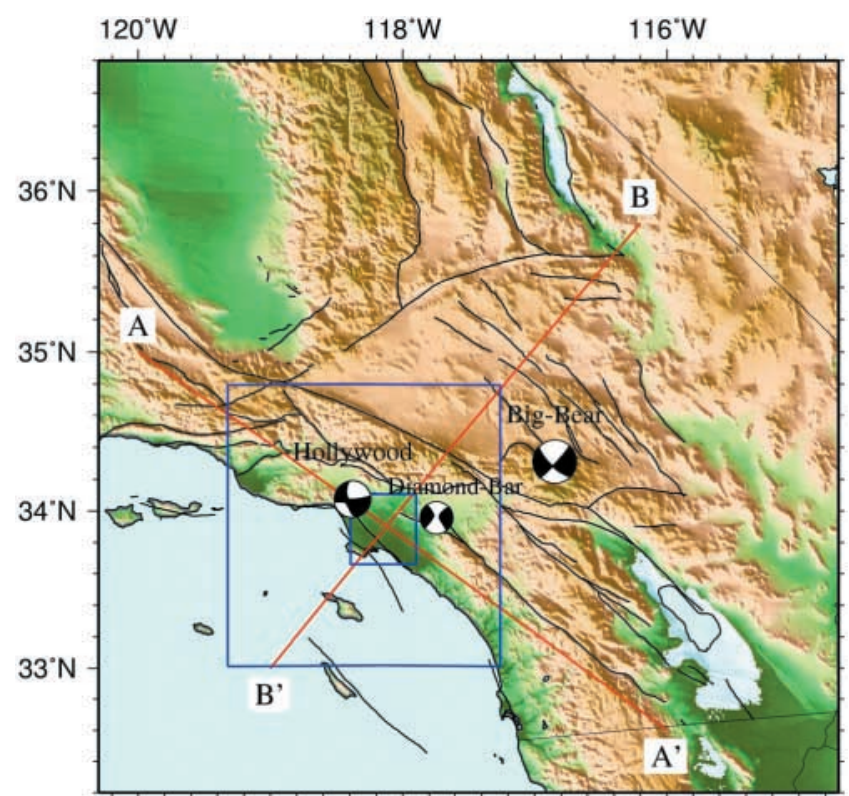

(a)
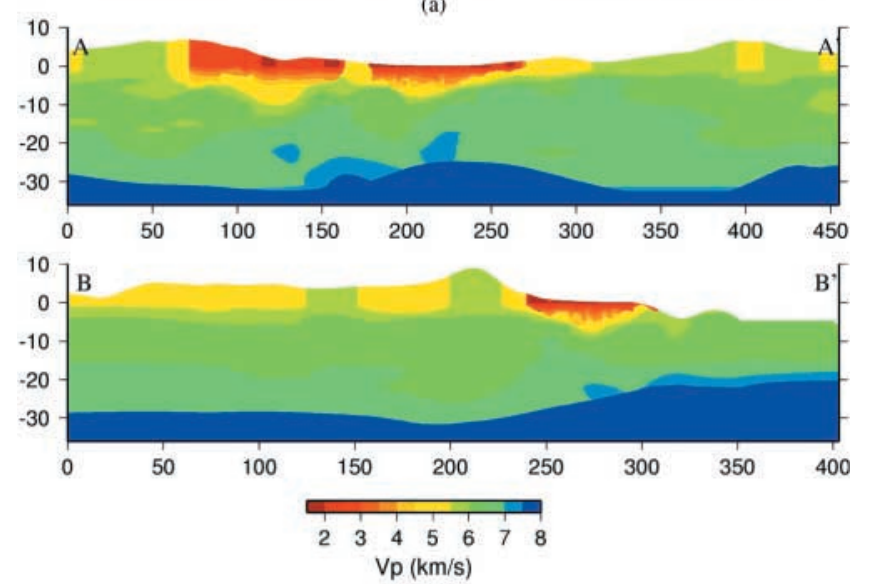

(b)

Figure 1. (a) Topographic map with shaded relief of southern California showing the extent of our 3D integrated velocity model. The largest blue box indicates the area covered by the medium-resolution GOCAD model developed by Süss and Shaw (2003), which includes the Ventura and San Fernando basins, the San Gabriel Mountains, part of the Mojave, and the Peninsular Ranges. The smaller blue box indicates the high-resolution part of the GOCAD model centered on the Los Angeles basin. The epicentral locations and source mechanisms of the three earthquakes discussed in this article are denoted by beachballs. The major late Quaternary faults (Jennings, 1975) are also displayed. (b) Compressional-wave speed variations along two cross sections AA' and $\mathrm{BB}^{\prime}$. Cross section $\mathrm{AA}^{\prime}$ runs from the Coast Ranges through the Ventura and Los Angeles basins to the Peninsular Ranges. Notice the significant 3D wave-speed variations, and in particular the very low wave-speed sedimentary basins. Also notice the shallow Moho underneath the Los Angeles basin. Cross section BB' runs from Death Valley through the Mojave and San Gabriel Mountains to the Los Angeles basin. Note again the low wave-speeds underneath Los Angeles and the shallow Moho under the continental borderland. This cross section also highlights the substantial topography and bathymetry (exaggerated 5 times) that is incorporated in our 3D model. 
jub et al., 2003; Komatitsch et al., 2004). The method combines the geometric flexibility of the finite-element method with an accurate representation of the wave field in terms of high-degree Lagrange polynomials. It is straightforward to incorporate surface topography and bathymetry, as well as topography on any internal discontinuities, into the spectralelement mesh. Because of the use of Lagrange polynomials and Gauss-Lobatto-Legendre quadrature, the mass matrix is exactly diagonal, which makes it relatively simple to implement the method on parallel computers (Komatitsch et al., 2003). Attenuation is accommodated by introducing memory variables (Komatitsch and Tromp, 1999). We use a constant shear quality factor of 90 within the basin and no attenuation elsewhere (Komatitsch et al., 2004). Our calculation of synthetics as well as Fréchet derivatives for earthquakes in southern California is based upon this method, which is described in detail by Komatitsch et al. (2004). The combination of a detailed crustal model and a very accurate numerical technique results in generally good fits between data and synthetic seismograms on all three components of most stations in the SCSN network at periods of $6 \mathrm{sec}$ and longer (Komatitsch et al., 2004). This provides us with reliable 3D Green's functions for the retrieval of source parameters.

\section{Moment Tensor Inversion Theory}

Suppose we have $N$ observed time series $\left\{d_{i}(t) ; i=1\right.$, $\ldots, N\}$ and $N$ corresponding synthetic seismograms $\left\{s_{i}(t\right.$, $\mathbf{m}) ; i=1, \ldots, N\}$ to constrain a point source model $\mathbf{m}=$ $\left\{m_{\mathrm{j}} ; j=1, \ldots, n\right\}$. In the case of an inversion for the six elements of the moment tensor $\mathbf{M}=\left\{M_{r r}, M_{\theta \theta}, M_{\phi \phi}, M_{r \theta}\right.$, $\left.M_{r \phi}, M_{\theta \phi}\right\}$, we have $n=6$ and $\mathbf{m}=\{\mathbf{M}\}$. An inversion for the moment tensor $\mathbf{M}$ and event depth $d_{\mathrm{s}}$ involves $n=7$ and $\mathbf{m}=\left\{\mathbf{M}, d_{\mathrm{s}}\right\}$. An inversion for the moment tensor $\mathbf{M}$ and the event location $\mathbf{r}_{s}$ results in $n=9$ and $\mathbf{m}=\left\{\mathbf{M}, \mathbf{r}_{s}\right\}$. Although theoretically and numerically feasible, the period range used in our simulations does not enable us to invert for the source half-duration of small- to moderate-size earthquakes.

Given a set of source model parameters $\mathbf{m}$, the misfit between the data and the synthetics may be assessed based upon the least-square waveform misfit function

$$
E_{1}(\mathbf{m})=\frac{1}{2 A_{1}} \sum_{i=1}^{N} w_{i} \int\left[d_{i}(t)-s_{i}(t, \mathbf{m})\right]^{2} d t .
$$

$A_{1}$ is a normalization factor, which can take the value of $\frac{1}{2} \sum_{i=1}^{N} w_{i} \int d_{i}(t)^{2} d t$ to ensure that $E_{1}(\mathbf{m})$ generally takes values between zero and one. Weights $w_{i}=w_{i}^{a} w_{i}^{d} w_{i}^{c}, i=$ $1, \ldots, N$ may be assigned based upon azimuth-related, $w_{i}^{a}$, epicentral distance-related, $w_{i}^{d}$, and component-related criteria, $w_{i}^{c}$. The disadvantage of the waveform misfit function (1) is that it is susceptible to cycle skips (i.e., the synthetic is advanced or delayed by one wave cycle or more relative to the data). An alternative misfit function may be defined based upon the envelope of the waveforms. For example, the data envelope function is defined by

$$
e\left(d_{i}(t)\right)=\left[d_{i}^{2}(t)+\hat{d}_{i}^{2}(t)\right]^{1 / 2}
$$

where $\hat{d}_{i}(t)$ is the Hilbert transform of $d_{i}(t)$. The corresponding envelope misfit function is

$$
E_{2}(\mathbf{m})=\frac{1}{2 A_{2}} \sum_{i=1}^{N} w_{i} \int\left[e\left(d_{i}(t)\right)-e\left(s_{i}(t, \mathbf{m})\right)\right]^{2} d t,
$$

where $A_{2}=\frac{1}{2} \sum_{i=1}^{N} w_{i} \int e\left(d_{i}(t)\right)^{2} d t$, and $E_{2}(\mathbf{m})$ usually takes values between zero and one. The disadvantage of the envelope misfit function (3) is that it does not provide any phase information, but when combined with the waveform misfit function (1), cycle skips may be avoided.

It is often desirable to subject the inversion to certain constraints. For example, if we require the moment tensor to have zero trace, that is, the source involves no change in volume, then the inversion needs to be subject to the constraint $C_{1}(\mathbf{m})=m_{1}+m_{2}+m_{3}=0$. If we further require that the earthquake is representable by a double-couple source mechanism, the moment tensor is subject to the additional constraint $C_{2}(\mathbf{m})=\operatorname{det}(\mathbf{M})=0$.

Combining all these ingredients, we minimize the following misfit function

$$
\begin{aligned}
E\left(\mathbf{m}, \mu_{1}, \mu_{2}\right)=\lambda E_{1}(\mathbf{m})+ & (1-\lambda) E_{2}(\mathbf{m}) \\
+ & \mu_{1} C_{1}(\mathbf{m})+\mu_{2} C_{2}(\mathbf{m}),
\end{aligned}
$$

where $\lambda$ and $1-\lambda$ denote the relative weights of the waveform misfit function $E_{1}(\mathbf{m})$ and the envelope misfit function $E_{2}(\mathbf{m})$, and $\mu_{1}$ and $\mu_{2}$ are the Lagrange multipliers associated with the constraints $C_{1}(\mathbf{m})$ and $C_{2}(\mathbf{m})$. The optimal source parameters $\mathbf{m}^{\text {new }}$ may be determined by solving the set of $n$ equations

$$
\frac{\partial E}{\partial m_{j}}\left(\mathbf{m}, \mu_{1}, \mu_{2}\right)=0, \quad j=1, \ldots, n,
$$

subject to the constraints $C_{1}(\mathbf{m})=0$ when $\mu_{1}$ is nonzero and $C_{2}(\mathbf{m})=0$ when $\mu_{2}$ is nonzero. Under certain assumptions, equation (5) can always be reduced to a linear system. For the detailed formulation of both linear and nonlinear inversions, please refer to the Appendix.

\section{Time Shifts}

The theory presented in the previous section is based on the assumption that we have perfect knowledge of the velocity structure in the region. Therefore, the differences between data and synthetics for given source parameters are 
completely due to errors in these parameters. Obviously this assumption does not hold in general. Source and structural effects are always entangled, and the difference between data and synthetics is due to both errors in the source mechanism and the inadequate $3 \mathrm{D}$ model. $3 \mathrm{D}$ velocity variations cause advances or delays in the arrival times of seismic phases as well as changes in the associated waveforms. Introducing the envelope misfit function (3) may help prevent cycle skip problems caused by relatively modest travel-time anomalies; but for large velocity variations, such as those in southern California, the envelope function may not be sufficiently effective.

Komatitsch et al. (2004) illustrate that differences between the true 3D velocity structure and our 3D model mainly affect the arrival times of seismic phases but do not significantly alter the associated waveforms at periods of $6 \mathrm{sec}$ and longer. Therefore, we can shift the synthetics with respect to the data to obtain the optimal correlation between the two, and then attribute differences in waveforms between the data and synthetics to the source parameters of the earthquake. This approach was demonstrated to be quite useful in accommodating effects due to 3D heterogeneity for the purpose of source-mechanism inversions by Zhao and Helmberger (1994). However, it may not be applicable to certain stations if the velocity variations between the source and the stations are not well described by our 3D velocity model, for example, when waveforms are severely distorted. Therefore, careful selection of stations is required to select paths for which our 3D velocity model produces decent waveforms compared to the data. Of course the whole procedure requires an initial source model $\mathbf{m}^{0}$ for which we calculate the synthetics for our 3D model. By correlating the data $d_{i}(t)$ and synthetics $s_{i}\left(t, \mathbf{m}^{0}\right)$ we obtain the time lag $\delta t_{i}$ between the two that gives

$$
\max _{\delta t_{i}} \frac{\int d_{i}(t) s_{i}\left(t-\delta t_{i}, \mathbf{m}^{0}\right) d t}{\left[\int d_{i}(t)^{2} d t \int s_{i}\left(t-\delta t_{i}, \mathbf{m}^{0}\right)^{2} d t\right]^{1 / 2}}
$$

We then apply the same time lag to all the Fréchet derivatives and invert for source model parameters by minimizing a waveform misfit function with time lags:

$$
\begin{aligned}
E_{\mathrm{s}}(\mathbf{m})=\frac{1}{2 A_{1}} & \sum_{i=1}^{N} w_{i} \int\left[d_{i}(t)-s_{i}\left(t-\delta t_{i}, \mathbf{m}^{0}\right)\right. \\
& \left.-\sum_{j} \frac{\partial s_{i}}{\partial m_{j}}\left(t-\delta t_{i}, \mathbf{m}^{0}\right)\left(m_{j}-m_{j}^{0}\right)\right]^{2} d t .
\end{aligned}
$$

This is the preferred misfit function in our inversion procedure. Another advantage of using equation (7) is that it is not affected by small timing errors associated with either the instrument or the origin time of the event. We will evaluate how well our synthetics for the new source parameters $\mathbf{m}^{\text {new }}$ fit the data by calculating the variance reduction

$$
\sigma_{i}=\frac{\int\left[d_{i}(t)-s_{i}\left(t-\delta t_{i}, \mathbf{m}^{\text {new }}\right)\right]^{2} d t}{\int d_{i}(t)^{2} d t}
$$

Alternatively, we may determine how much the misfit function (7) has been reduced after the inversion by calculating the misfit reduction:

$$
\chi=\left[E_{s}\left(\mathbf{m}^{0}\right)-E_{s}\left(\mathbf{m}^{\text {new }}\right)\right] / E_{s}\left(\mathbf{m}^{0}\right) .
$$

Fortunately, for most earthquakes with $M_{\mathrm{W}} \geq 3.5$ we can use source-mechanism estimations from other methods as initial solutions. However, there are cases in which we do not have other solutions to start with, meaning we do not have any reference from which to either select the data or obtain time lags to shift synthetics and Fréchet derivatives. Nevertheless, we can always determine our own initial source mechanism based upon a preliminary moment-tensor-only linear inversion in which we select data traces solely based upon their signal-to-noise ratio. As we show in later examples, this inversion procedure proves to be quite robust for determining the source mechanisms of small- and moderate-size earthquakes in southern California and can be easily implemented automatically whenever an earthquake occurs.

\section{Numerical Tests}

In order to solve (5), we need to calculate the derivatives of the synthetics with respect to the source parameters to obtain the linear system (A5) or (A16) as given in the Appendix. In our case, this implies numerically differentiating synthetics with respect to the source parameters. Because the synthetics $s_{j}(t, \mathbf{m})$ are linear combinations of the moment tensor elements, the derivatives $\frac{\partial s_{j}}{\partial m_{i}}(t), i=1, \ldots, 6$ can be easily obtained by running forward calculations for moment tensors that have a nonzero $i$ th element while all the other elements are zero. However, $s_{j}(t, \mathbf{m})$ is a nonlinear function of depth, latitude, and longitude, $\left\{m_{i}, i=7,8,9\right\}$, therefore, in order to approximate the derivatives for these three parameters with finite differences, appropriate intervals for the finite-difference length should be chosen such that the derivatives of the misfit function remain relatively constant within these intervals. Furthermore, in order to understand the functional dependence of the misfit function on depth, latitude, and longitude for (1) as well as (3) and (7), we perform several synthetic tests for the 9 September 2001, $M_{\mathrm{W}} 4.2$ Hollywood event (see Fig. 1 for its location and mechanism). We adopt the event location and mechanism obtained by the Thio and Kanamori (1995) method $\left(34.0745^{\circ} \mathrm{N}, 118.3792^{\circ} \mathrm{W}\right.$, and a depth of $5.4 \mathrm{~km}$; refer to Table 1 for further details), calculate synthetics at all available SCSN stations inside our 3D model, and regard these as synthetic data. We then run simulations for a series of test source models, generate synthetics at SCSN stations, and evaluate the misfit function between the synthetic data and 
Table 1

Source Parameters of the Hollywood Event for Various Inversion Methods or Parameter Settings

\begin{tabular}{lccccc}
\hline Type & $\mathbf{M}_{\mathrm{w}}$ & Strike/Dip/Rake & Depth & $\varepsilon$ & Mech. \\
\hline Moment + location & 4.08 & $173 / 74 / 182$ & 5.6 & 0.13 & $\boldsymbol{\Theta}$ \\
Moment + depth & 4.08 & $171 / 72 / 179$ & 5.5 & 0.13 & $\boldsymbol{\Theta}$ \\
Moment only & 4.08 & $171 / 73 / 179$ & 5.4 & 0.13 & $\boldsymbol{\Theta}$ \\
Double couple & 4.08 & $172 / 73 / 178$ & 5.5 & 0.00 & $\boldsymbol{\Theta}$ \\
Surface wave only & 4.08 & $173 / 74 / 182$ & 5.6 & 0.13 & $\boldsymbol{\Theta}$ \\
Equal weight & 4.09 & $174 / 75 / 182$ & 5.6 & 0.14 & $\boldsymbol{\Theta}$ \\
Thio and Kanamori (1995) & 4.07 & $171 / 66 / 183$ & 5.4 & 0.04 & $\boldsymbol{\Theta}$ \\
Zhu and Helmberger (1996) & 4.17 & $168 / 74 / 159$ & 6.0 & 0.00 & $\boldsymbol{\Theta}$ \\
\hline
\end{tabular}

The first six rows are the mechanisms determined by our inversions, and the last two rows are the inversion results based upon the methods of Thio and Kanamori (1995) and Zhu and Helmberger (1996). For simplicity, only one of the fault plane solutions is listed in each row. Strike, dip, and rake are in degrees, and depth is in kilometers. The parameter $\varepsilon$ represents the magnitude of the non-double-couple component in a moment tensor (Dziewonski et al., 1981; Lay and Wallace, 1995).

the synthetics for each test source model. The misfit values have all been normalized by the amplitude of the data such that a misfit value of 1 means that all the synthetics fit the data on average as poorly as a straight line, while a misfit value of 0 means that all the synthetics fit the data perfectly. For all the synthetic tests and inversions presented in this paper, both data and synthetics have been bandpass filtered between $6 \mathrm{sec}$ and $40 \mathrm{sec}$.

\section{Synthetic Test: Latitude and Longitude}

In order to examine how the waveform misfit function without time shifts (1) and the waveform misfit function with shifts (7) vary as a function of latitude, we fix all remaining eight source parameters, varying only the latitude from $34.06^{\circ} \mathrm{N}$ to $34.08^{\circ} \mathrm{N}$ by $0.0025^{\circ}$ increments, and calculate the synthetics for each case. We then evaluate the misfit functions (1) and (7). In Figure 2a, the dashed and the solid lines, respectively, show how the waveform misfit function with and without time shifts varies with latitude. The functions have both symmetry and convexity centered on the true latitude $34.0745^{\circ} \mathrm{N}$. Notice that the waveform misfit function with time shifts (dashed line) has values that are about onethird of those for the waveform misfit function without time shifts (solid line). The difference becomes larger when the latitude moves farther away from the true latitude. This suggests that the difference in waveform measured by (7) only takes up a small portion of the difference between the data and synthetics measured by (1) in the case of an incorrect latitude, and thus the arrival time difference should be responsible for a large portion of the waveform misfit without time shifts. Therefore, the sensitivity of the misfit function (7) to latitudinal variations will be smaller compared to (1). All the above statements hold true for longitudinal variations as well. Notice that within a latitudinal interval of $0.0025^{\circ}$, the derivative of the misfit function remains about constant, therefore we are confident that this should give a good ap- proximation to the latitudinal derivatives at the initial solution.

\section{Synthetic Test: Depth}

We vary the depth from $4 \mathrm{~km}$ to $9.2 \mathrm{~km}$ by $0.4 \mathrm{~km}$ increments and fix all other eight parameters to study the functional dependence of the misfit functions (1) and (7) on depth. As one can see from Figure 2b, both misfit functions change significantly faster at shallower depths compared to greater depths, suggesting that both misfit functions are more sensitive to shallower earthquakes, which may be due to the more rapidly varying shallow structure. Notice that the values for a waveform misfit function with time shifts (dashed line) are only slightly smaller than those for a waveform misfit function without time shifts (solid line), showing that the depth variation of earthquakes mainly gives rise to changes in waveform but not in the travel time for waves at periods of $6 \mathrm{sec}$ and longer. This means that we should have good resolution for event depth when we use the waveform misfit function with time shifts (7) to determine the optimal source parameters. Also notice that within an interval of $0.4 \mathrm{~km}$, the derivative of the misfit function remains almost constant, therefore it should be a good approximation to obtain the depth derivatives at the initial solution.

\section{Synthetics Test: Envelope Misfit versus Waveform Misfit without Time Shifts}

As mentioned earlier, the existence of structural heterogeneity that is not part of our 3D velocity model is the most severe problem that we need to overcome in our source inversions. One way of doing this, in the case of relatively small 3D heterogeneity, is to use a combination of waveform misfit without time shifts and the envelope misfit, as in (4). To demonstrate that the envelope misfit function does help accommodate the effects of heterogeneity not included in the $3 \mathrm{D}$ model, we assign random time shifts of up to $3 \mathrm{sec}$ 


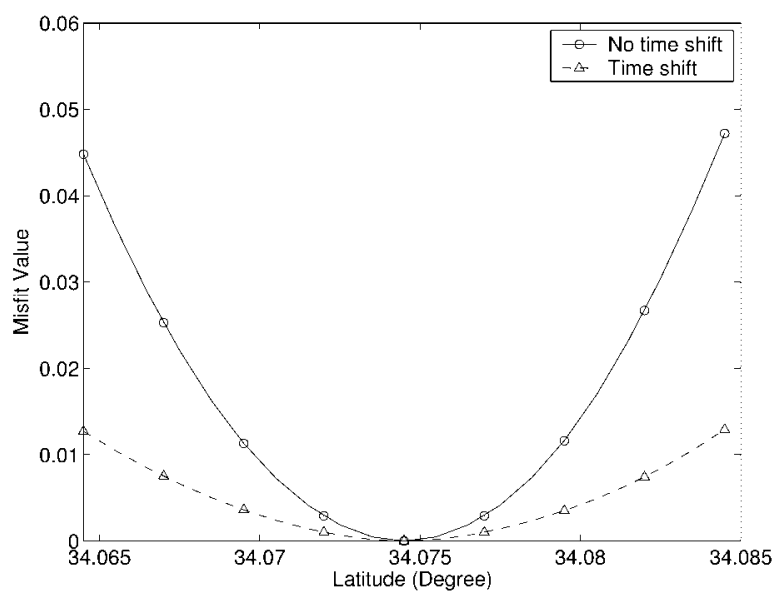

(a)

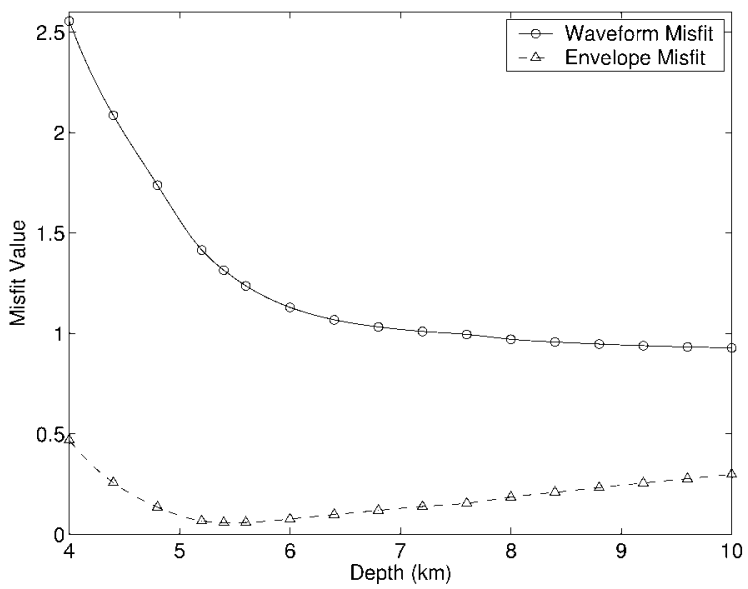

(c)

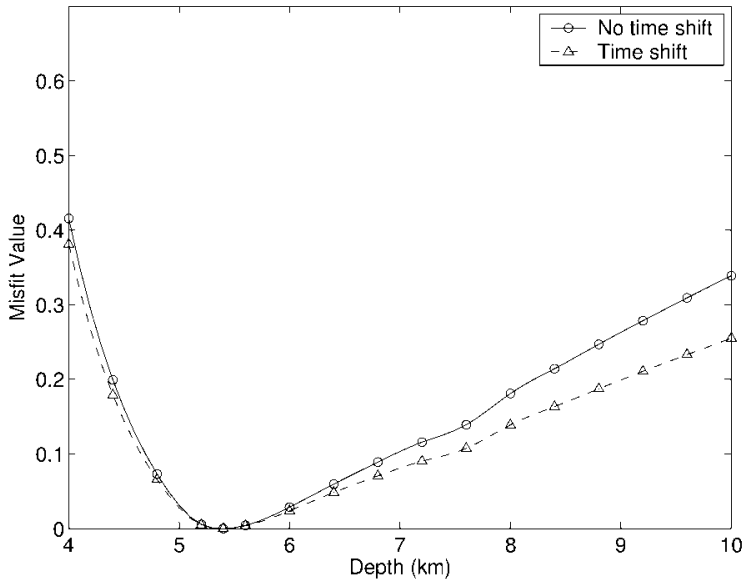

(b)

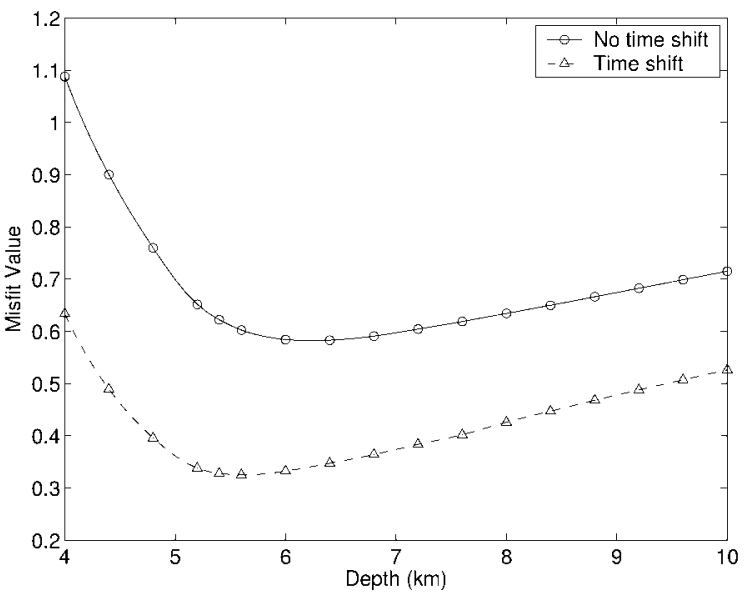

(d)

Figure 2. (a) Waveform misfit with or without time shifts between the synthetic data and the synthetics as a function of latitude. (b) Waveform misfit with or without time shifts between the synthetic data and the synthetics as a function of depth. (c) Waveform misfit without time shifts and envelope misfit between the shifted synthetic data and the synthetics as a function of depth. (d) Waveform misfit with and without time shifts between actual data and the synthetics as a function of depth.

to the synthetic data to mimic the effect of 3D heterogeneity and regard the shifted synthetic data as our data. We evaluate both the waveform misfit without time shifts (1) and the envelope misfit (3) between this data and synthetics for each depth calculated in the previous section. We show the functional dependence of both misfit functions on depth in Figure 2c. Notice that because of the simulated 3D heterogeneity, the waveform misfit function without time shifts, which calculates directly the difference between data and synthetics, does not have its minimum centered on the true depth of $5.4 \mathrm{~km}$. However, the envelope misfit function, which is not very sensitive to small arrival-time anomalies, still has its minimum at $5.4 \mathrm{~km}$. This suggests that in the case of relatively small unmodeled heterogeneity (for example, in global moment tensor inversions), we may use a combination of the envelope misfit function (3) and the waveform misfit function without time shifts (1) to recover source mechanisms. However, as shown by Komatitsch et al. (2004), the travel-time anomalies for surface waves at periods of $6 \mathrm{sec}$ and longer may be as large as $5 \mathrm{sec}$ for stations at distances of $250 \mathrm{~km}$ and larger in our 3D southern California model. Therefore, allowing time shifts for the waveform misfit function is a better choice to invert for earthquake source parameters in southern California. Of course as soon as we have an improved shear-velocity model for the region we should be able to dispense with these time shifts.

\section{Data Test: Depth}

Next, we obtain actual data recorded at various SCSN stations for the 9 September 2001 Hollywood event using the STP program provided by the Southern California Earthquake Data Center. We calculate the waveform misfit function with and without time shifts for selected data traces and 
corresponding synthetics for different depths (refer to the Hollywood Earthquake section for details about the selection criteria). The result is shown in Figure 2d. Global minima are achieved by both misfit functions at around $5.5-6.5 \mathrm{~km}$; however, the misfit function without time shifts (solid line) is flatter around the minimum compared to the misfit function with time shifts (dashed line), which has a distinct minimum at about $5.6 \mathrm{~km}$. We suspect that unmodeled 3D effects may have obscured the depth minimum of the misfit function without time shifts. Also notice that the value of the misfit function with time shifts is typically $50 \%$ smaller than the value of the misfit function without time shifts, suggesting again that the arrival-time anomaly takes up a significant portion of the misfit between data and synthetics. Of course the situation can be more complicated when all the moment tensor elements and location parameters vary. Nevertheless, the waveform misfit function with time shifts has very good sensitivity to the depth of the earthquake and is used in our inversion process.

\section{Inversion Procedures and Technical Details}

\section{Calculation and Processing of Synthetics}

If we are able to obtain an initial source solution for an earthquake from either first-motion or other inversion methods, we can calculate the Fréchet derivatives of the synthetics with respect to all nine source parameters, including the six moment tensor elements, depth, latitude, and longitude of the earthquake. The SEM calculations involve mesh with a total of 45.4 million grid points (i.e., 136 million degrees of freedom) and require 14 gigabytes of distributed memory. Therefore it takes, in total, about one day to calculate all the derivatives needed for our source inversion. To save computation time, we combine the moment tensor Fréchet derivatives to obtain the synthetics for the initial solution using (A1). We then pad up to $20 \mathrm{sec}$ of zeroes before the first arrival to all the synthetics and Fréchet derivatives and bandpass-filter them between $6 \mathrm{sec}$ and $40 \mathrm{sec}$.

\section{Data Processing}

We collect all the broadband BH component data for the earthquake using the STP program, as mentioned earlier. We correct the raw data for the instrument response to obtain displacement records using the poles-and-zeroes files provided by the SCSN network and then apply the same filters as applied to the synthetics.

\section{Inversion}

We select those data traces whose corresponding synthetics have relatively decent waveform fits to perform our source inversion. As we will discuss in more detail, we require that the time shift between data and synthetics $\delta t_{i}$ that satisfies (6) be less than a prescribed value that varies with distance, while requiring that the best correlation value itself be large enough to guarantee good similarity between data and synthetics. Since the correlation values are always normalized by the amplitude of the data and synthetics, we also require that the amplitude ratio of the data and synthetics be smaller than a certain value, to avoid problems associated with the stations themselves.

Regional $P_{\mathrm{nl}}$ waves have been shown to be relatively insensitive to 3D heterogeneity in the crust (Helmberger and Engen, 1980), therefore the $P_{\mathrm{nl}}$ phase is a good candidate to invert for the source. We split the vertical and radial components of the data and synthetic traces into a $P_{\mathrm{nl}}$ part and a Rayleigh surface-wave part for stations at distances larger than $150 \mathrm{~km}$. Since the $P_{\mathrm{nl}}$ waves are relatively small in amplitude compared to the surface waves, we also implement a weighting factor for $P_{\mathrm{nl}}$ waves to balance their contribution in the inversion.

We add the contributions of all the selected traces to form the matrix equation (A5) or (A16), which has dimension less than or equal to 11 . We can simply solve this linear system by Gaussian elimination with partial pivoting (Conte and Boor, 1980) to obtain the new source parameters $\mathbf{m}^{\text {new }}$. In general, the initial source parameters are close to the true source parameters, therefore we do not iterate the process. We evaluate the variance reduction (8) of the synthetics for the new source parameters $\mathbf{m}^{\text {new }}$ relative to the data at each station to examine how well the new synthetics fit the data. We also evaluate how much the waveform misfit function with time shifts has been reduced by $\mathbf{m}^{\text {new }}$ compared to the initial source parameters $\mathbf{m}^{0}$ by going over all the selected data and synthetics again and calculating the misfit reduction (9). We are also interested in evaluating (9) for each trace, whether it has been used in the inversion or not, to give us some idea of how much the waveform fits are improved by changing the source parameters.

In the following three sections we analyze three recent southern California earthquakes and determine their source parameters by the procedures outlined earlier. We also discuss the effects of velocity heterogeneity that is not part of our 3D model on the determination of earthquake source parameters.

\section{Hollywood Earthquake}

We first consider the 9 September $2001 M_{\mathrm{L}} 4.2$ Hollywood earthquake. This well-recorded event is small enough that the finite size of the source can be safely ignored. We obtain data from 125 stations within our model domain, with the nearest station at a distance of $7.7 \mathrm{~km}$ and the farthest at a distance of $348 \mathrm{~km}$. We use initial source parameters determined by the method of Thio and Kanamori (1995) (the second-from-last entry in Table 1). To show that this initial solution is accurate enough to start with, we plot in Figure 3 the correlation value (6) between the vertical components of the data and the synthetics for this initial solution at most of the SCSN broadband stations. Stations with high correlation values are shown in warm colors, while stations with low correlation values are shown in cold colors. We see, 


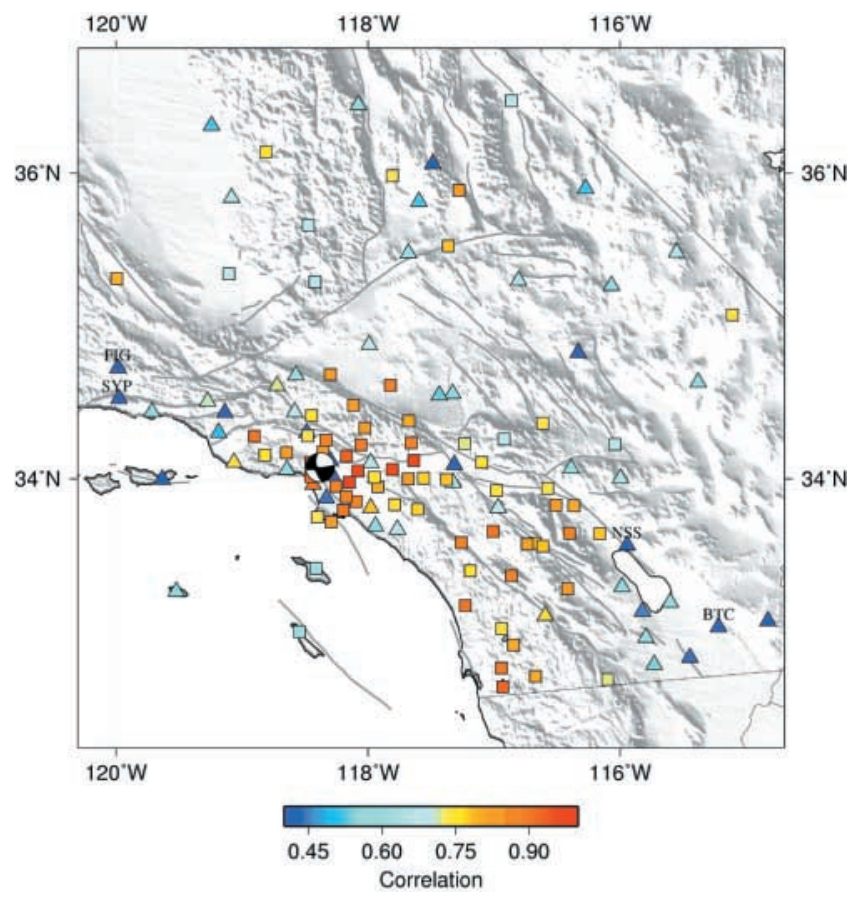

Figure 3. Correlation of the vertical component data and synthetics for the initial solution at most of the SCSN broadband stations for the Hollywood event. Warm colors indicate high similarity of the waveforms, while cold colors may indicate regions where our 3D velocity model does not describe the true structure adequately. Squares indicate stations whose vertical component is used in the source inversion. All the data and synthetics have been filtered between $6 \mathrm{sec}$ and $40 \mathrm{sec}$. The epicenter of the Hollywood earthquake is indicated by a black star.

overall, very good waveform fits throughout southern California, as also observed by Komatitsch et al. (2004). We find that, in general, with increasing distance the correlation values decrease because of the smaller signal-to-noise ratio at more remote stations. As discussed in Komatitsch et al. (2004), the Salton trough and Santa Barbara and Ventura basin regions are filled with thick sediments. However, they are outside the Süss and Shaw (2003) model range, and the background tomographic model (Hauksson, 2000) is not particularly good at describing the surface geology in these locations. Therefore, stations in these regions generally have small correlation values (Fig. 3, station NSS, BTC, FIG, SYP, etc.). In any event, this initial solution is good enough to help us select the good data traces to further fine-tune the source parameters. We use the whole seismogram (up to 3 min) and cut the vertical and radial traces into $P_{\mathrm{nl}}$ waves (from $(-5.0+$ dist $) / 7.8 \mathrm{sec}$ to $t_{\mathrm{s}}+2.0 \mathrm{sec}$, where dist denotes the distance of the station from the epicenter of the earthquake in kilometers and $t_{\mathrm{s}}$ denotes the predicted arrival time for $S$ waves using the standard southern California model [Hadley and Kanamori, 1977; Dreger and Helmberger, 1990]), and surface waves (from $(-15.0+$ dist $) / 3.5$ sec to $(35.0+$ dist $) / 3.1 \mathrm{sec})$ at distances larger than $150 \mathrm{~km}$.
We then shift the synthetics and Fréchet derivatives with respect to the corresponding data traces or segments to obtain the best correlation. We select $P_{\mathrm{nl}}$ data traces by requiring that the time shift between data and the initial synthetics be less than $3.0+$ dist $/ 80.0 \mathrm{sec}$, the amplitude ratio be less than 2.5, and the correlation be larger than 0.70 . We select Love- and Rayleigh-wave data by requiring that the time shift between data and the initial synthetics be less than (3.0 + dist) $/ 50.0 \mathrm{sec}$, the amplitude ratio be less than 2.5 , and the correlation be larger than 0.65 . These values for cutting and selecting are empirical, but they have proven to be robust and appropriate for earthquakes in southern California. In total we selected for our inversion $28 P_{\mathrm{nl}}$ traces from vertical and radial seismograms, and 209 surface-wave traces from all three components, including 71 vertical traces, 93 transverse traces, and 53 radial traces. We set a relative weight of 3:1 for $P_{\mathrm{nl}}$ and surface-wave traces to account for their amplitude difference. We taper the data, synthetics, and Fréchet derivatives by a trapezoidal function centered around the maximum value of the synthetics. We also apply weights $w=N_{\mathrm{a}}^{-a}$ (dist) ${ }^{-d} N_{\mathrm{c}}^{-c}$ to every single trace, where $N_{\mathrm{a}}$ represents the number of traces selected in the same azimuthal bin as the trace under consideration; $a$ is an exponent we set to express how much we want to emphasize differences in station azimuth; $d$ is set to be 1.13 for $P_{\mathrm{nl}}$ traces, 0.74 for Rayleigh waves, and 0.55 for Love waves to accommodate an empirical amplitude decay with distance for southern California (Zhu and Helmberger, 1996); $N_{c}$ is the number of traces selected with the same component as the current trace; and $c$ is a factor that controls the relative weights of the three components. Since the original SCSN station distribution is not uniform in azimuth, there may be directions in which the stations are sparse while the model is not well constrained, in which case a too large value of $a$ would contaminate the final inversion result with contributions from stations with less-reliable 3D Green's functions. Therefore we generally set $a$ to a small value. Because typically the transverse and vertical components of the synthetics are fitted better than the radial component, we set $c$ to a smaller value for the radial component than the other two components.

To check the robustness of our inversion procedures, we usually invert with several combinations of parameters. Table 1 shows the inversion results for the following cases: (1) invert for both moment tensor and location; (2) invert for both moment tensor and depth; (3) invert for moment tensor only; (4) same as (1) but constrain the moment tensor to be a double couple; (5) same as (1) except that only surface-wave traces are used in the inversion; (6) same as (1) except that no weights are applied to the traces. For comparison, the solution obtained by the methods of Thio and Kanamori (1995) and Zhu and Helmberger (1996) are also listed in the table. We consider case 2 separately from case 1 , because typical mislocations of earthquakes in southern California are about 1-2 km in both the latitudinal and longitudinal directions, while they can be as large as $3-4 \mathrm{~km}$ in 
depth (Hauksson, 2000), and we are more concerned with obtaining a better depth. Also, because our misfit function has greater sensitivity to depth than to latitude and longitude, it is desirable to invert only for the moment tensor and depth to check for the stability of the inversion process. Comparing the inversion results in Table 1, all results are similar to the initial solution, with the strike and rake within $2^{\circ}$ and the dip within $9^{\circ}$. Although the presence of a non-double-couple component in zero-trace inversions may reduce the misfit, we believe it is not necessarily well resolved, and may just reflect imperfections in our 3D Green's function. The inversion results show that the depth of this earthquake is about $5.6 \pm 0.2 \mathrm{~km}$. Notice that this depth is a little shallower than that obtained by the method of Zhu and Helmberger (1996), therefore the focal mechanism and the moment magnitude are slightly different from those obtained by their method as well. Our evaluation of misfit reduction (9) is about $13 \%$ for the selected $P_{\mathrm{nl}}$ traces and 7\% for all selected surface-wave traces.

\section{Big Bear Mainshock}

We also study the 22 February $2003 M_{\mathrm{L}} 5.4$ Big Bear mainshock. This is the biggest event in southern California since the year 2000. It occurred in the San Bernardino Mountains, which are surrounded by complicated fault systems (Fig. 1). We apply the same data selection criteria as in the previous section, except that we allow the time shift between the data and synthetics to be larger to accommodate a possible offset in origin time. Also, because this is a larger earthquake than the Hollywood event, we set the correlation criteria values to be a little larger than in the previous section. Data is available for 137 stations in our model region. From this data set, we selected $51 P_{\mathrm{nl}}$ traces and 169 surface-wave traces, which include 66 vertical traces, 89 transverse traces, and 63 radial traces. The fact that we use fewer surface-wave records than for the Hollywood event is not surprising, since this event occurred outside the model of Süss and Shaw (2003), and we expect the overall waveform fits to be worse than for the Hollywood event. Although our integrated model has the highest resolution in the Los Angeles basin, the waves might have already developed complications before propagating into the basin.

The inversion results are summarized in Table 2. Figure 4 shows the variance reduction given by ( 8 ) for the surfacewave part on the transverse component at each station to demonstrate how well the synthetics for the inversion result (moment tensor and location) fit the data. Obviously the variance reduction for each trace is related to both the correlation value as well as the amplitude difference between the data and the synthetics. Figure 4 shows that most stations have quite large variance reductions (50\%). By comparing to Figure 5, which shows the waveform fits of the surface waves for some of the typical stations in southern California, it is clear that stations in the nodal directions of the transverse component tend to have smaller variance reductions because of the relatively small signal-to-noise ratio. However, our misfit function (7) recognizes the absolute amplitude information in the data, therefore the stations in the nodal directions also provide critical information in estimating the source parameters as long as the noise level is not too high. Notice that we still have low variance reductions in the Salton trough and Santa Barbara and Ventura basin areas. Our variance reduction in the Los Angeles basin is relatively small, which may be due to complications in the waveforms developed along the propagation path. Overall, we achieve significant variance reduction for traces that have been used in the inversion (indicated by squares). The difference in variance reduction at different stations may also reflect the fact that a significant portion of the waveform misfit is due to complicated 3D velocity structure. Different inversion schemes show that the depth of the earthquake is about $6.4 \pm 0.2 \mathrm{~km}$.

\section{Diamond Bar Earthquake}

The 14 December 2001, $M_{\mathrm{L}}$ 4.0 Diamond Bar event is a unique event in that it occurred close to the Los Angeles basin and had a deep hypocenter of $14 \mathrm{~km}$ (from the SCSN earthquake catalog). Besides its significant depth, its small

Table 2

Source Parameters for the Big Bear Earthquake

\begin{tabular}{|c|c|c|c|c|c|}
\hline Type & $\mathbf{M}_{\mathrm{w}}$ & Strike/Dip/Rake & Depth & $\varepsilon$ & Mech. \\
\hline Moment + location & 4.92 & $134 / 72 / 186$ & 6.3 & 0.04 & 0 \\
\hline Moment + depth & 4.92 & $134 / 72 / 186$ & 6.3 & 0.04 & 8 \\
\hline Moment only & 4.92 & $134 / 72 / 187$ & 6.3 & 0.03 & 8 \\
\hline Double couple & 4.92 & $134 / 72 / 186$ & 6.3 & 0.00 & 8 \\
\hline Surface wave only & 4.92 & $134 / 71 / 186$ & 6.3 & 0.04 & 8 \\
\hline Equal weight & 4.93 & $134 / 72 / 187$ & 6.3 & 0.03 & 8 \\
\hline Thio and Kanamori (1995) & 4.93 & $320 / 75 / 200$ & 6.3 & 0.00 & 8 \\
\hline Zhu and Helmberger (1996) & 4.96 & $135 / 72 / 193$ & 6.5 & 0.00 & Q \\
\hline
\end{tabular}

See Table 1 for explanation. 
Big Bear Earthquake

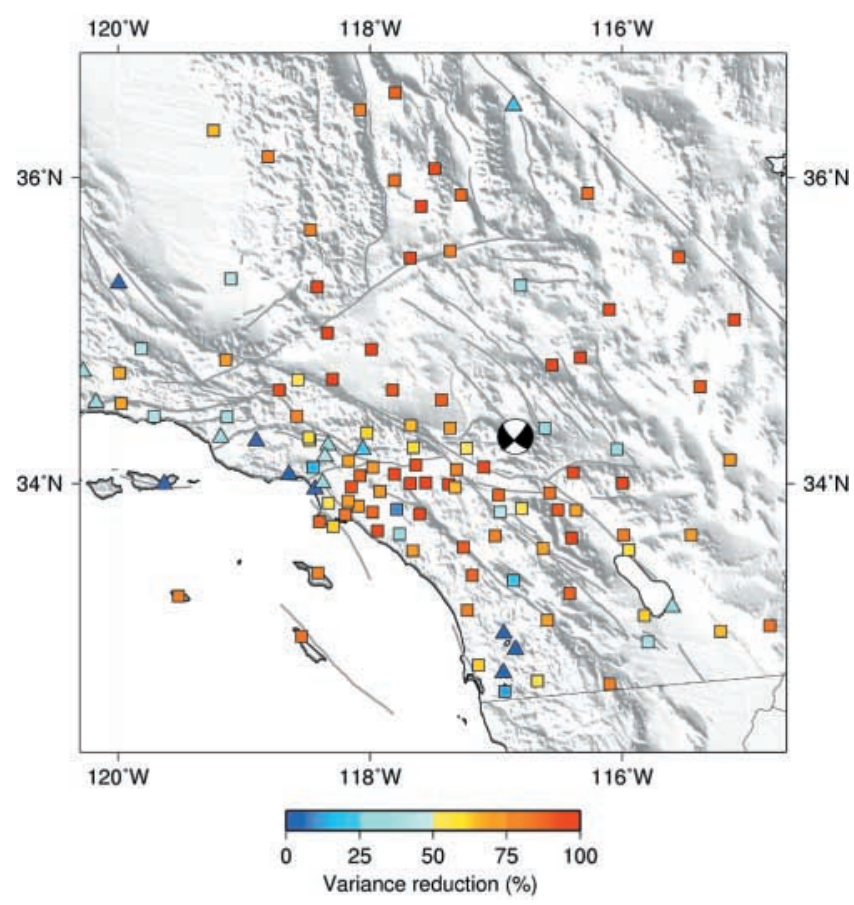

Figure 4. Variance reduction for transverse component synthetics calculated based upon the inverted source parameters at most of the SCSN broadband stations for the 2003 Big Bear mainshock. Warm colors indicate large variance reductions, where the new synthetics generally fit the data better than at stations indicated by the cold colors. Squares indicate stations whose transverse component is used in the source inversion. Only stations with a correlation larger than 0.4 are plotted. The epicenter and source mechanism of the earthquake are denoted by the beachball.

magnitude also makes the data rather noisy to invert for a stable mechanism. The method of Thio and Kanamori (1995) is not able to produce a stable solution because their method mostly uses stations outside the basin, which have relatively smaller signal-to-noise ratios for this earthquake. It is therefore desirable that we produce an initial solution based upon our own preliminary inversion. We select data traces based upon the noise level before the origin time, that is, the signal-to-noise ratio. Since we have a rough estimate of the magnitude of this earthquake, we require the amplitude of the data to be below a certain value to avoid problems such as station clipping or long-period noise. Based upon these criteria, we selected 123 traces for the inversion, including 31 vertical traces, 60 transverse traces, and 32 radial traces. Figure 6 shows the station distribution of the selected traces (both red and green triangles) on the transverse component. Notice that they are well distributed in both distance and azimuth, showing the good quality of the data as well as the robustness of the data selection. As a preliminary inversion, we use only the surface-wave part of the traces. We apply no shift to the derivatives, because we do not have

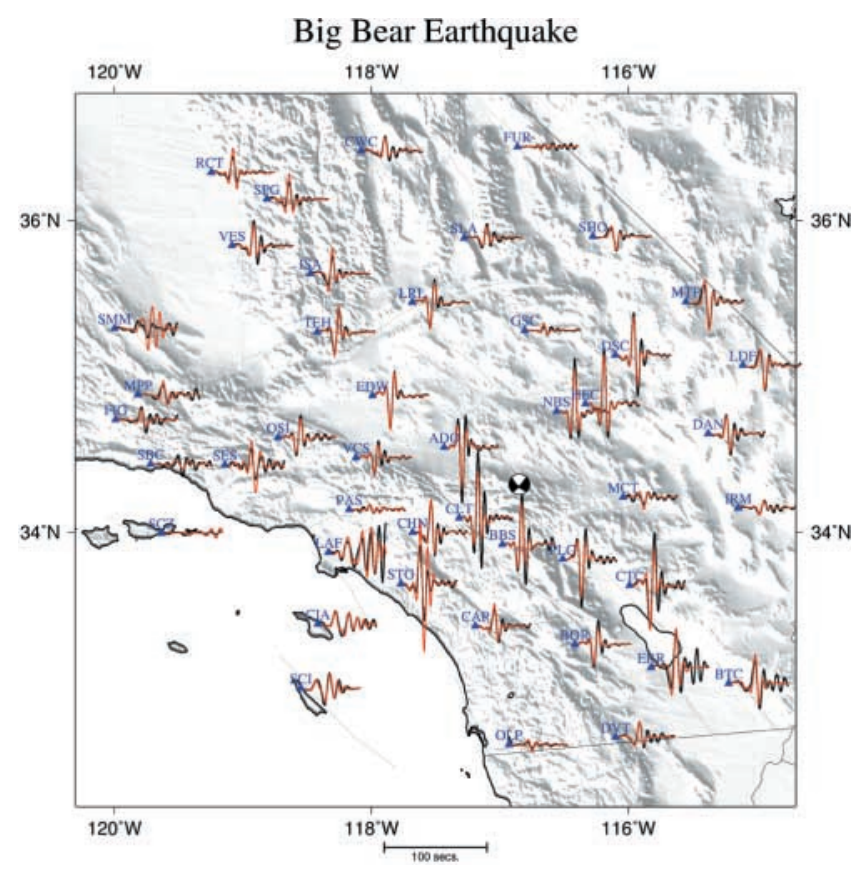

Figure 5. Selected waveform fits for transverse component data (black) and synthetics (red) for the inverted source parameters of the Big Bear mainshock. The synthetics have been shifted with respect to the data to obtain the best correlation. Combined with Figure 4, it illustrates the general fits between data and synthetics throughout southern California. More examples of waveform fits can be found in Komatitsch et al. (2004).

synthetics to obtain the $\delta t_{i}$ value given by (6) for each trace, which means that we minimize the waveform misfit function without time shifts (1). We take the network event depth, $14 \mathrm{~km}$, and invert only for a zero-trace moment tensor, which is a linear inversion that does not require any initial source mechanism. The inversion result gives us a source mechanism of $324^{\circ}$ strike, $88^{\circ}$ dip, and $178^{\circ}$ rake, a moment magnitude of 3.64, and a variance reduction of the new synthetics (without time shifts) with respect to the selected data traces of $27 \%$. Obviously, when no time shifts are allowed, the quality of the inversion might be compromised due to problems such as cycle skips.

We next use the preliminary inversion result as an initial solution, calculate the location derivatives with respect to this initial solution as described in the Numerical Tests section and perform the standard source inversion to minimize the waveform misfit function with time shifts. The stations for which transverse component data were selected are plotted in Figure 6 (both red and blue triangles). Notice more than half of the selected data overlap with the data selected in the preliminary inversion, showing the suitability of the initial selection procedures. The well-distributed coverage of the selected data traces also gives us confidence in the inversion results we present in Table 3. Different inversion schemes show that the event depth changed from $14 \mathrm{~km}$ to about $12.0 \pm 0.5 \mathrm{~km}$. Figure 7 demonstrates the misfit re- 


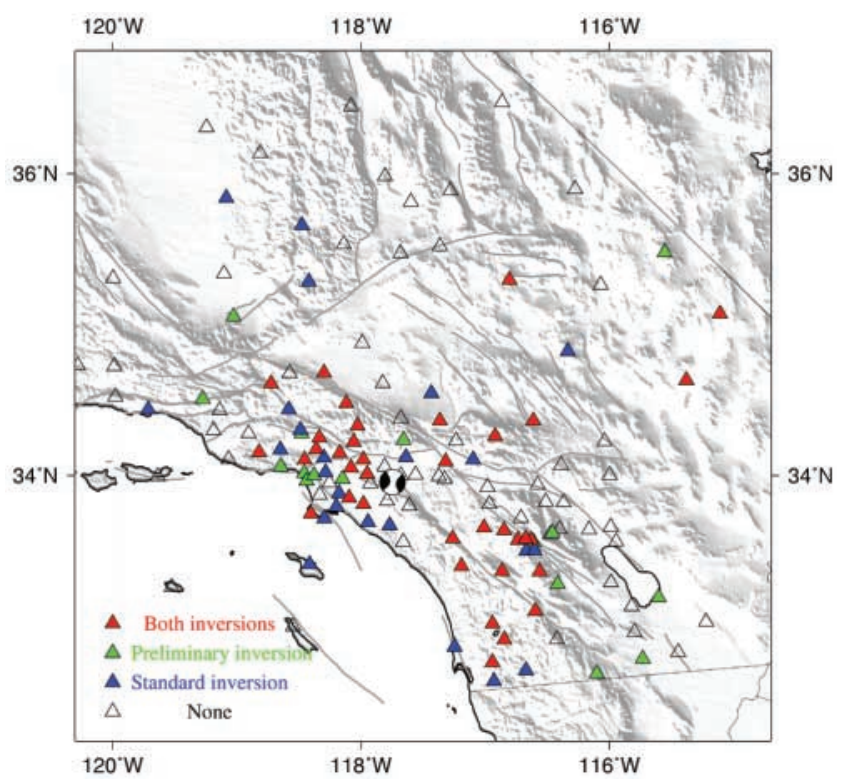

Figure 6. Stations whose transverse component has been used in the preliminary source inversion and the standard source inversion for the Diamond Bar event. Red triangles indicate stations that are used in both inversions, green triangles indicate stations used only in the preliminary inversion, blue triangles indicate stations used only in the second inversion, and white triangles indicate stations used in neither inversion.

duction for stations with correlations higher than 0.4. Most of the stations that were used (denoted by squares) have a misfit reduction of at least $20 \%$. Even some stations that are not used in the inversion have positive misfit reduction values, showing that the second inversion has significantly reduced the misfit function compared to the initial result. We achieve a total misfit reduction of about $18 \%$ for the selected $P_{\mathrm{nl}}$ traces and $25 \%$ for the surface-wave traces.

\section{Discussion}

We typically use about 200 traces to invert for the source parameters of earthquakes with $M_{\mathrm{W}} \geq 4$.0, and about 120 traces for a $M_{\mathrm{W}} 3.7$ earthquake. Therefore, we may be able to extend our inversion method to earthquakes with $M_{\mathrm{W}}$ $\geq 3.5$ for southern California. In the Los Angeles basin, where we have the highest model resolution, we may be able to push the limit to even smaller earthquakes $\left(M_{\mathrm{W}} 3.3\right)$. The inversion procedure described for the Hollywood, Big Bear mainshock, and Diamond Bar earthquakes can be very easily automated for events with $M_{\mathrm{W}} \geq 3.5$ in southern California. The point-source mechanism should be quite a good representation for these small- and moderate-sized earthquakes. By minimizing the waveform misfit function with time shifts, we can accommodate the effects of an imperfect 3D Green's function on the source inversion reasonably well. Future improvements to the 3D velocity model should render such time shifts unnecessary.

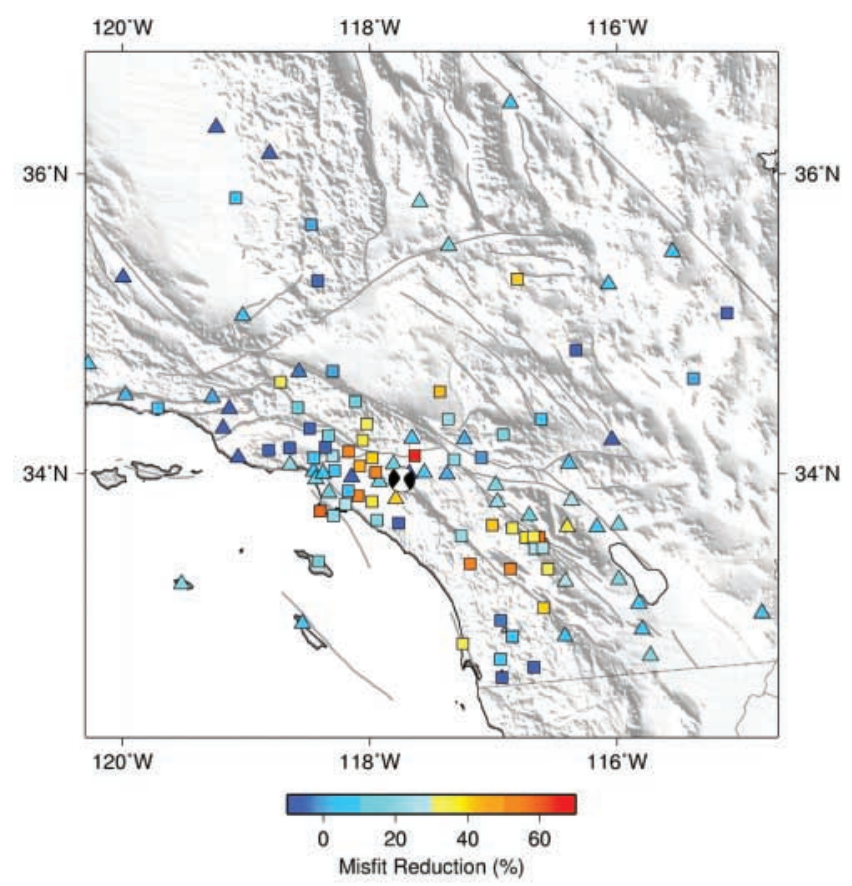

Figure 7. Misfit reduction for transverse component seismograms by the source parameters from the standard inversion compared to the ones determined by a preliminary inversion at most of the SCSN stations for the Diamond Bar event. Only stations with correlations larger than 0.4 are plotted. Squares indicate stations whose transverse component is used in the source inversion. Warm colors indicate a large misfit reduction, where the improvement in the source mechanism helps to explain the misfit between the data and the synthetics for the initial solution, whereas cold colors indicate locations where the waveform misfit is mainly due to an imperfect 3D Green's function.

\section{Conclusion}

We determined source parameters for three southern California earthquakes based upon spectral-element simulations of regional seismic wave propagation in an integrated 3D southern California velocity model. By minimizing the waveform misfit function with time shifts, which accommodates the imperfect 3D Green's function, we were able to determine moment tensors and locations for these earthquakes. The inversion results agree very well with estimates from either body- or surface-wave inversion methods for the Hollywood and Big Bear earthquakes. The inversion procedures can be performed with or without an initial solution, as shown for the Diamond Bar earthquake. This enables us to perform automatic source parameter inversions for $M_{\mathrm{W}}$ $\geq 3.5$ earthquakes in southern California. Since there are about 40 earthquakes with $M_{\mathrm{L}} \geq 3.5$ in southern California every year, we will be able to build and maintain an earthquake source mechanism database, which should be of importance for understanding local fault systems and tectonic processes. 
Table 3

Source Parameters for the Diamond Bar Earthquake

\begin{tabular}{lccccc}
\hline Type & $\mathbf{M}_{\mathrm{w}}$ & Strike/Dip/Rake & Depth & $\varepsilon$ & Mech. \\
\hline Moment + location & 3.69 & $320 / 90 / 187$ & 12.0 & 0.15 & \\
Moment + depth & 3.69 & $320 / 88 / 186$ & 11.9 & 0.15 & \\
Moment only & 3.72 & $320 / 89 / 183$ & 14.0 & 0.15 & \\
Double couple & 3.70 & $321 / 84 / 188$ & 11.5 & 0.00 & $\mathbf{8}$ \\
Surface wave only & 3.69 & $320 / 90 / 187$ & 12.0 & 0.15 & 0.14 \\
Equal weight & 3.69 & $320 / 88 / 189$ & 12.0 & 0.16 & $\mathbf{8}$ \\
Preliminary inversion & 3.64 & $324 / 88 / 178$ & 14.0 & \\
\hline
\end{tabular}

See Table 1 for explanation. Our preliminary inversion result is listed as the initial solution used in the second inversion.

\section{Acknowledgments}

We thank Egill Hauksson and Ying Tan for providing first-motion and body-wave moment tensor mechanisms; Hiroo Kanomori, Egill Hauksson, Donald V. Helmberger, Gene A. Ichinose, and an anonymous reviewer for helpful comments; and SCSN for access to the data. All the map figures were produced with GMT (Wessel and Smith, 1991). This research was funded in part by the National Science Foundation under grant EAR0309576 and the USGS NEHRP. This research was also supported by the Southern California Earthquake Center (SCEC). SCEC is funded by NSF Cooperative Agreement EAR-0106924 and USGS Cooperative Agreement 02HQAG0008. The SCEC contribution number for this paper is 772. This is contribution No. 9080 of the Division of Geological and Planetary Sciences of the California Institute of Technology.

\section{References}

Chaljub, E., Capdeville, and J. P. Vilotte (2003). Solving elastodynamics in a fluid-solid heterogeneous sphere: a parallel spectral element approximation on non-conforming grids, J. Comput. Phys. 187, 457491.

Conte, S. D., and C. Boor (1980). Elementary Numerical Analysis: An Algorithm Approach, Third Ed., McGraw-Hill, New York.

Dreger, D. S., and D. V. Helmberger (1990). Broadband modeling of local earthquakes, Bull. Seism. Soc. Am. 80, 1162-1179.

Dreger, D. S., and D. Helmberger (1991). Source parameters of the Sierra Madre earthquake from regional and local body waves, Geophys. Res. Lett. 18, 2015-2018.

Dziewonski, A. M., T. A. Chou, and J. H. Woodhouse (1981). Determination of earthquake source parameters from waveform data for studies of global and regional seismicity, J. Geophys. Res. 86, 2825-2852.

Gilbert, F., and A. M. Dziewonski (1975). An application of normal mode theory to the retrival of structural parameters and source mechanisms from seismic spectra, Phil. Trans. R. Soc. London, Ser. A 278, 187269.

Hadley, D., and H. Kanamori (1977). Seismic structure of the Transverse Ranges, California, Geol. Soc. Am. Bull. 88, 1469-1478.

Hauksson, E. (2000). Crustal structure and seismicity distribution adjacent to the Pacific and North America plate boundary in Southern California, J. Geophys. Res. 105, 13,875-13,903.

Hauksson, E., L. Jones, S. Perry, and K. Hutton (2002). Emerging from the stress shadow of the $1992 M_{\mathrm{W}} 7.3$ Landers southern California earthquake? A preliminary assessment, Seism. Res. Lett. 73, 33-38.

Helmberger, D. V., and G. R. Engen (1980). Modeling the long-period body waves from shallow earthquakes at regional ranges, Bull. Seism. Soc. Am. 70, 1699-1714.

Jennings, P. (1975). Fault map of California with volcanoes, thermal springs and thermal wells at 1:750,000 scale, in Geological Data Map
1, California Division of Mines and Geology, Sacramento, California. Komatitsch, D., and J. Tromp (1999). Introduction to the spectral-element method for 3-D seismic wave propagation, Geophys. J. Int. 139, 806822 .

Komatitsch, D., and J. Tromp (2000a). Spectral-element simulations of global seismic wave propagation: I. Validation, Geophys. J. Int. 149, 390-412.

Komatitsch, D., and J. Tromp (2000b). Spectral-element simulations of global seismic wave propagation: II. 3-D models, oceans, rotation, and self-gravitation, Geophys. J. Int. 150, 303-318.

Komatitsch, D., and J. P. Vilotte (1998). The spectral-element method: an efficient tool to simulate the seismic response of 2D and 3D geological structures, Bull. Seism. Soc. Am. 88, 368-392.

Komatitsch, D., S. Tsuboi, J. Chen, and J. Tromp (2003). A 14.6 billion degrees of freedom, 5 teraflops, 2.5 terabyte earthquake simulation on the Earth Simulator, in Proc. of the ACM/IEEE Supercomputing SC 2003 Conference, November 2003.

Komatitsch, D., Q. Liu, J. Tromp, P. Süss, C. Stidham, and J. H. Shaw (2004). Simulations of ground motion in the Los Angeles basin based upon the spectral-element method, Bull. Seism. Soc. Am. 94, 187206.

Lay, T., and T. C. Wallace (1995). Modern Global Seismology, Academic Press, New York.

Mallet, J. L. (1992). Discrete smooth interpolation in geometric modeling, Computer-Aided Design 24, 178-191.

Ritsema, J., and T. Lay (1993). Rapid source mechanism determination of large $\left(M_{\mathrm{W}} \geq 5\right)$ earthquakes in the western United States, Geophys. Res. Lett. 20, 1611-1614.

Süss, M. P., and J. H. Shaw (2003). $P$-wave seismic velocity structure derived from sonic logs and industry reflection data in the Los Angeles basin, California, J. Geophys. Res. 108, 2170, doi 10.1029/ 2001JB001628.

Thio, H. K., and H. Kanamori (1995). Moment-tensor inversions for local earthquakes using surface waves recorded at TERRAscope, Bull. Seism. Soc. Am. 95, 1021-1038.

Wallace, T. C., and D. V. Helmberger (1981). A technique for the inversion of regional data in source parameter studies, J. Geophys. Res. 86, 1679-1685.

Wessel, P., and W. H. F. Smith (1991). Free software helps map and display data, EOS Trans. AGU 72 (441), 445-446.

Zhao, L., and D. V. Helmberger (1994). Source estimation from broadband regional seismograms, Bull. Seism. Soc. Am. 84, 91-104.

Zhu, L., and D. V. Helmberger (1996). Advancement in source estimation techniques using broadband regional seismograms, Bull. Seism. Soc. Am. 86, 1634-1641.

Zhu, L., and H. Kanamori (2000). Moho depth variation in southern California from teleseismic receiver functions, J. Geophys. Res. 105, 2969-2980. 


\section{Appendix: Spectral-Element Moment-Tensor Inversion Theory}

Starting from the misfit function (4), we can either perform linear or nonlinear source inversions depending on our choice of source parameters and constraints. The waveform misfit function with time shifts follows the same formulation, except that a time lag must be applied to both the synthetics and the Fréchet derivatives. For simplicity, we set the normalization factors $A_{1}$ and $A_{2}$ to one.

\section{Linear Inversions}

If we set $\lambda=1$ (i.e., we do not include the envelope misfit function), $\mu_{2}=0$ (i.e., we do not require a doublecouple solution), and invert only for the six elements of the moment tensor by holding the earthquake location fixed, then the problem becomes linear. In this case the synthetics $s_{i}(t, \mathbf{m})$ can be expressed as linear combinations of the Fréchet derivatives with respect to moment tensor elements $\frac{\partial s_{i}}{\partial m_{k}}(t)$ :

$$
s_{i}(t, \mathbf{m})=\sum_{k=1}^{6} \frac{\partial s_{i}}{\partial m_{k}}(t) m_{k} .
$$

Substitution of (A1) into (4) yields

$$
\begin{aligned}
\frac{\partial E_{1}}{\partial m_{j}}+ & \mu_{1} \frac{\partial C_{1}}{\partial m_{j}}= \\
& -\sum_{i=1}^{N} w_{i} \int\left[d_{i}(t)-\sum_{k=1}^{6} \frac{\partial s_{i}}{\partial m_{k}}(t) m_{k}\right] \frac{\partial s_{i}}{\partial m_{j}}(t) d t \\
& +\mu_{1} \frac{\partial C_{1}}{\partial m_{j}}=0 .
\end{aligned}
$$

If we define

$$
\begin{aligned}
H_{j k} & =\sum_{i=1}^{N} w_{i} \int \frac{\partial s_{i}}{\partial m_{k}}(t) \frac{\partial s_{i}}{\partial m_{j}}(t) d t, \\
G_{j} & =\sum_{i=1}^{N} w_{i} \int d_{i}(t) \frac{\partial s_{i}}{\partial m_{j}}(t) d t,
\end{aligned}
$$

then (A2) becomes

$$
\sum_{k=1}^{6} H_{j k} m_{k}-G_{j}+\mu_{1} \frac{\partial C_{1}}{\partial m_{j}}=0
$$

Let $\mathbf{U}=\left\{\partial C_{1} / \partial m_{j} ; j=1, \ldots, 6\right\}=[1,1,1,0,0,0]^{T}$, where a superscript $T$ denotes the transpose, then (A4) becomes the following matrix equation:

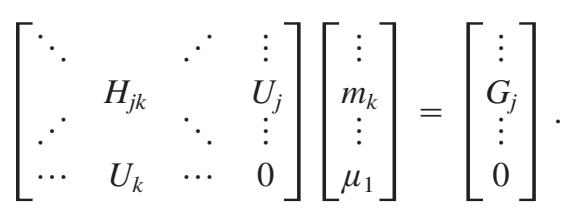

This equation can be solved for the six elements of the moment tensor. Notice that $\left(H_{j k}\right)$ is a positive-definite symmetric matrix. Therefore, when the zero-trace constraint is not invoked, a unique solution is guaranteed to exist.

\section{Nonlinear Inversions}

When $\lambda \neq 1$ or $\mu_{2} \neq 0$, equation (4) becomes nonlinear with respect to $\mathbf{m}$, either because we need to invert for the event location or because the derivative of the envelope misfit function is nonlinear. In any event, an initial solution $\mathbf{m}^{0}$ is required to solve (4). In our case we use point-source parameters inverted using alternative methods (Thio and Kanamori, 1995; Zhu and Helmberger, 1996) as the starting solution. The synthetics can be linearized with respect to the initial source parameters:

$s_{i}(t, \mathbf{m})=s_{i}\left(t, \mathbf{m}^{0}\right)+\sum_{k=1}^{n} \frac{\partial s_{i}}{\partial m_{k}}\left(t, \mathbf{m}^{0}\right)\left(m_{k}-m_{k}^{0}\right)$,

and (5) becomes

$$
\begin{aligned}
\lambda \frac{\partial E_{1}}{\partial m_{j}}\left(\mathbf{m}^{0}\right)+(1 & -\lambda) \frac{\partial E_{2}}{\partial m_{j}}\left(\mathbf{m}^{0}\right)+\mu_{1} \frac{\partial C_{1}}{\partial m_{j}}\left(\mathbf{m}^{0}\right) \\
& +\mu_{2} \frac{\partial C_{2}}{\partial m_{j}}\left(\mathbf{m}^{0}\right)=0, j=1, \ldots, n
\end{aligned}
$$

In the following we consider the case of a double-couple source inversion in detail; other nonlinear inversions have similar characteristics. Using (A6) and assuming that the initial solution is sufficiently close to the true solution, we obtain

$$
\begin{aligned}
\frac{\partial E_{1}}{\partial m_{j}}\left(\mathbf{m}^{0}\right) & =-\sum_{i=1}^{N} w_{i} \int\left[d_{i}(t)-s_{i}\left(t, \mathbf{m}^{0}\right)\right. \\
& \left.-\sum_{k=1}^{n} \frac{\partial s_{i}}{\partial m_{k}}\left(t, \mathbf{m}^{0}\right)\left(m_{k}-m_{k}^{0}\right)\right] \frac{\partial s_{i}}{\partial m_{j}}\left(t, \mathbf{m}^{0}\right) d t, \\
\frac{\partial E_{2}}{\partial m_{j}}\left(\mathbf{m}^{0}\right) & =-\sum_{i=1}^{N} w_{i} \int\left[e\left(d_{i}(t)\right)-e\left(s_{i}\left(t, \mathbf{m}^{0}\right)\right)\right. \\
& \left.-\sum_{k=1}^{n} \frac{\partial e\left(s_{i}\right)}{\partial m_{k}}\left(t, \mathbf{m}^{0}\right)\left(m_{k}-m_{k}^{0}\right)\right] \frac{\partial e\left(s_{i}\right)}{\partial m_{j}}\left(t, \mathbf{m}^{0}\right) d t,
\end{aligned}
$$

where 


$$
\begin{aligned}
\frac{\partial e\left(s_{i}\right)}{\partial m_{j}}\left(t, \mathbf{m}^{0}\right)=e\left(s_{i}\left(t, \mathbf{m}^{0}\right)\right)^{-1 / 2} & {\left[s_{i}\left(t, \mathbf{m}^{0}\right) \frac{\partial s_{i}}{\partial m_{j}}\left(t, \mathbf{m}^{0}\right)\right.} \\
& \left.+\hat{s}_{i}\left(t, \mathbf{m}^{0}\right) \frac{\partial \hat{s}_{i}}{\partial m_{j}}\left(t, \mathbf{m}^{0}\right)\right],
\end{aligned}
$$

and $\hat{s}(t, \mathbf{m})$ denotes the Hilbert transform of $s(t, \mathbf{m})$. Let

$$
\begin{aligned}
H_{j k} & =\sum_{i=1}^{N} w_{i} \int\left[\lambda \frac{\partial s_{i}}{\partial m_{k}}\left(t, \mathbf{m}^{0}\right) \frac{\partial s_{i}}{\partial m_{j}}\left(t, \mathbf{m}^{0}\right)\right. \\
& \left.+(1-\lambda) \frac{\partial e\left(s_{i}\right)}{\partial m_{k}}\left(t, \mathbf{m}^{0}\right) \frac{\partial e\left(s_{i}\right)}{\partial m_{j}}\left(t, \mathbf{m}^{0}\right)\right] d t \\
G_{j} & =\sum_{i=1}^{N} w_{i} \int\left\{\lambda\left[d_{i}(t)-s_{i}\left(t, \mathbf{m}^{0}\right)\right] \frac{\partial s_{i}}{\partial m_{j}}\left(t, \mathbf{m}^{0}\right)\right. \\
& \left.+(1-\lambda)\left[e\left(d_{i}(t)\right)-e\left(s_{i}\left(t, \mathbf{m}^{0}\right)\right)\right] \frac{\partial e\left(s_{i}\right)}{\partial m_{j}}\left(t, \mathbf{m}^{0}\right)\right\} d t,
\end{aligned}
$$

and rewrite $(\mathrm{A} 7)$ as

$$
\begin{aligned}
f_{j}\left(\mathbf{m}, \mu_{1}, \mu_{2}\right)=\sum_{k=1}^{n} H_{j k}\left(m_{k}-m_{k}^{0}\right)-G_{j} \\
\quad+\mu_{1} \frac{\partial C_{1}}{\partial m_{j}}(\mathbf{m})+\mu_{2} \frac{\partial C_{2}}{\partial m_{j}}(\mathbf{m})=0, j=1, \ldots, n,
\end{aligned}
$$

with constraints $f_{n+1}(\mathbf{m})=C_{1}(\mathbf{m})=0$ and $f_{n+2}(\mathbf{m})=$ $C_{2}(\mathbf{m})=0$. To solve the nonlinear equations (A11), we use the nonlinear Newton's iterative solver (Conte and Boor, 1980). Let us define a vector $\mathbf{x}=\left[\mathbf{m}, \mu_{1}, \mu_{2}\right]$ and an initial solution vector $\mathbf{x}^{0}=\left[\mathbf{m}^{0}, \mu_{1}^{0}, \mu_{2}^{0}\right]$ (we usually start with $\left.\mu_{1}^{0}=\mu_{2}^{0}=0\right)$, then the $(i+1)$ th iteration can be expressed as

$$
\frac{\partial \mathbf{f}}{\partial \mathbf{x}}\left(\mathbf{x}^{i}\right)\left(\mathbf{x}^{i+1}-\mathbf{x}^{i}\right)=-\mathbf{f}\left(\mathbf{x}^{i}\right)
$$

If we define

$$
\begin{aligned}
\mathbf{Y} & =\left(\frac{\partial^{2} C_{2}}{\partial m_{j} \partial m_{k}}\right) \\
& =\left[\begin{array}{cccccc}
0 & m_{3} & m_{2} & 0 & 0 & -2 m_{6} \\
m_{3} & 0 & m_{1} & 0 & -2 m_{5} & 0 \\
m_{2} & m_{1} & 0 & -2 m_{4} & 0 & 0 \\
0 & 0 & -2 m_{4} & -2 m_{3} & 2 m_{6} & 2 m_{5} \\
0 & -2 m_{5} & 0 & 2 m_{6} & -2 m_{2} & 2 m_{5} \\
2 m_{6} & 0 & 0 & 2 m_{5} & 2 m_{4} & -2 m_{1}
\end{array}\right] \\
\mathbf{V} & =\left\{\begin{array}{c}
\left.\mathrm{A} C_{2} / \partial m_{j} ; j=1, \ldots, 6\right\} \\
=
\end{array}\right. \\
& -\left[m_{2} m_{3}-m_{6}^{2}, m_{1} m_{3}-m_{5}^{2}, m_{1} m_{2}-m_{4}^{2},-2\left(m_{3} m_{4}\right.\right. \\
& \left.\left.-m_{5} m_{6}\right),-2\left(m_{2} m_{5}-m_{4} m_{6}\right),-2\left(m_{1} m_{6}-m_{4} m_{5}\right)\right]^{T}
\end{aligned}
$$

and

$$
\begin{gathered}
\mathbf{D}=\left(\frac{\partial f_{j}}{\partial x_{k}}\right)=\left[\begin{array}{ccccc}
\ddots & & \ddots & \vdots & \vdots \\
. & H_{j k}+\mu_{2} Y_{j k} & & U_{j} & V_{j} \\
\cdots & & \ddots & \vdots & \vdots \\
\cdots & U_{k} & \cdots & 0 & 0 \\
V_{k} & \cdots & 0 & 0
\end{array}\right] \\
j, k=1, \ldots, n,
\end{gathered}
$$

then (A12) becomes a linear system

$$
\mathbf{D}^{\mathbf{i}}\left(\mathbf{x}^{i+1}-\mathbf{x}^{i}\right)=-\mathbf{f}\left(\mathbf{x}^{i}\right)
$$

and the source model parameters $\mathbf{m}$ are determined by iterating this linear system with respect to $\mathbf{x}$.

Seismological Laboratory

California Institute of Technology

1200 East California Boulevard

Pasadena, California 91125

Manuscript received 26 February 2004 